\title{
Decreased exposure to sunitinib due to concomitant administration of ifosfamide: results of a phase I and pharmacokinetic study on the combination of sunitinib and ifosfamide in patients with advanced solid malignancies
}

\author{
P Hamberg,', N Steeghs², WJ Loos', D van de Biessen', M den Hollander², M Tascilar', J Verweij', \\ H Gelderblom ${ }^{2}$ and S Sleijfer' \\ 'Department of Medical Oncology, Erasmus University Medical Center Daniel Den Hoed Cancer Center, Rotterdam, The Netherlands; ${ }^{2}$ Department of \\ Medical Oncology, Leiden University Medical Center, Leiden, The Netherlands
}

\begin{abstract}
BACKGROUND: This study aimed to define the maximally tolerated dose (MTD) of sunitinib combined with two different infusion schedules of ifosfamide.

METHODS: Patients with advanced solid tumours, good performance score, good organ function, and no standard therapy available were eligible. Continuous once daily sunitinib, in escalating doses per cohort, was combined with ifosfamide, $9 \mathrm{~g} \mathrm{~m}^{-2}$ for 3 days or $6 \mathrm{~g} \mathrm{~m}^{-2}$ for 5 days, administered every 3 weeks. Pharmacokinetic (PK) and pharmacodynamic (PD) assessments were performed. RESULTS: With growth-factor support, the MTD of sunitinib combined with either ifosfamide schedule was $12.5 \mathrm{mg}$ in 32 patients enrolled. Neutropenia-related adverse events were dose-limiting toxicities. Sunitinib did not affect ifosfamide PK. Ifosfamide significantly decreased exposure to sunitinib and increased exposure to its metabolite, SUI2662. No consistent changes in PD parameters were observed.

CONCLUSION: With growth-factor support, the MTD of sunitinib with both ifosfamide schedules was $12.5 \mathrm{mg}$. Ifosfamide produced decreased sunitinib blood levels because of CYP3A induction. As PK interactions cannot explain the relatively low sunitinib doses that can be combined with ifosfamide, synergy in toxicity is likely. Whether this also holds true for anti-tumour activity needs to be further explored. British Journal of Cancer (2010) I 02, 1699- 1706. doi:I0.1038/sj.bjc.6605696 www.bjcancer.com

Published online 18 May 2010

(c) 2010 Cancer Research UK
\end{abstract}

Keywords: sunitinib; ifosfamide; pharmacokinetic; VEGF; drug-interaction; phase I

The introduction of tyrosine kinase inhibitors (TKIs) specifically inhibiting tumour-driving factors was accompanied with high expectations with regard to their activity against solid malignancies. Their single-agent activity in most tumour types is, however, modest, with obvious exceptions in tumours such as renal cell carcinoma (RCC) and gastrointestinal stromal tumours (GIST) (Verweij et al, 2004; Motzer et al, 2007).

One potential way to augment the activity of TKIs is to combine them with conventional cytotoxic agents. In particular, combinations of TKIs targeting the vascular endothelial growth factor (VEGF) pathway and conventional cytotoxic agents seem attractive, given several potential mechanisms that may yield synergistic anti-tumour effects. Vascular endothelial growth factor produced by tumour cells results in the formation of new vasculature that is abnormal in structure and more permeable than normal vasculature. This causes a high interstitial pressure within the tumour, hindering the penetration of drugs into

\footnotetext{
*Correspondence: Dr P Hamberg; E-mail: a.hamberg@erasmusmc.nl This paper was previously presented in part at the Annual Meeting of the American Society of Clinical Oncology 2009 and the Annual Meeting of the American Society for Clinical Pharmacology and Therapeutics 2010. Received 26 February 2010; revised 8 April 2010; accepted 21 April 2010; published online 18 May 2010
}

tumours (Boucher and Jain, 1992). Inhibition of VEGF-mediated effects has been shown to decrease intra-tumoural interstitial pressure, thereby enhancing the delivery of concomitantly administered drugs (Boucher and Jain, 1992; Heldin et al, 2004; Willett et al, 2004). Other mechanisms that may contribute to synergistic interaction between VEGF-pathway inhibitors and conventional cytotoxic drugs include prevention of endothelial progenitor cell mobilisation from the bone marrow induced by chemotherapy and decreased production of tumour factors conferring resistance against chemotherapy (Simakajornboon et al, 2001; Dias et al, 2002; Tran et al, 2002; Riedel et al, 2004; Shaked et al, 2008).

Sunitinib is a potent inhibitor of VEGF receptors (VEGFR) $1-3$, KIT, platelet-derived growth factor receptor- $\alpha$ and- $\beta$, and Fms-like tyrosine kinase-3 (Flt3), and is one of the first and most commonly used VEGFR-TKIs. It is currently registered for the treatment of advanced RCC and imatinib-refractory GIST (Demetri et al, 2006; Motzer et al, 2007) and is being explored for its anti-tumour activity in a wide range of other tumour types. A potential attractive agent to combine with sunitinib is ifosfamide, an alkylating agent with established activity against a similar wide range of tumour entities including breast cancer, testicular cancer, lung cancer, sarcomas, and central nervous system (CNS) tumours such as medulloblastomas. 
This study aimed to define the maximally tolerated dose (MTD) of ifosfamide combined with sunitinib. Two different infusion schedules of ifosfamide were explored. In addition, extensive pharmacokinetic (PK) and pharmacodynamic (PD) studies were conducted.

\section{PATIENTS AND METHODS}

\section{Patient selection}

Patients with histologically or cytologically confirmed advanced or metastatic solid tumours, for whom no standard therapy was available, with an Eastern Cooperative Oncology Group (ECOG) performance status $<2$, were eligible. Other inclusion criteria were evaluable or measurable disease according to RECIST version 1 (Therasse et al, 2000 ), age $\geqslant 18$ years, life expectancy $\geqslant 12$ weeks, adequate bone marrow (neutrophil count $\geqslant 1.5 \times 10^{9}$ cells per l; platelets $\geqslant 100 \times 10^{9}$ cells per l; and haemoglobin $\geqslant 6.0 \mathrm{mmoll}^{-1}$ ), liver (serum bilirubin $\leqslant 1.5 \times$ upper limit of normal (ULN) and serum ASAT and ALAT $\leqslant 2.5 \times$ ULN or, if liver metastases were present, $\leqslant 5 \times$ ULN), and renal function (serum creatinin $\leqslant 1.5 \times$ ULN and creatinine clearance $\geqslant 60 \mathrm{ml} \mathrm{min}^{-1}$ ), two functioning kidneys, systolic blood pressure $<150 \mathrm{~mm} \mathrm{Hg}$, and diastolic blood pressure $<90 \mathrm{~mm} \mathrm{Hg}$ (treatment with two anti-hypertensive drugs was allowed). Main exclusion criteria were history of cardiovascular disease, known HIV seropositivity, and signs or symptoms of CNS metastases.

The study was designed and conducted under the approval of appropriate institutional review boards (METC 2006-273 and CME 06-273) and in accordance with the principles embodied in the Declaration of Helsinki. Written informed consent was obtained from each participant.

\section{Study design and drug dosing, escalation, and administration}

Daily oral sunitinib was planned to be evaluated in three dose cohorts, $12.5 \mathrm{mg}, 25 \mathrm{mg}$, and $37.5 \mathrm{mg}$, in combination with a fixed dose of ifosfamide, according to one of the standard schedules of monotherapy ifosfamide: $9 \mathrm{~g} \mathrm{~m}^{-2}$ administered as 3-day continuous intravenous infusion (CIV) at 3-weekly intervals. After establishing the MTD of sunitinib with this dose and schedule of ifosfamide, this sunitinib dose was evaluated with ifosfamide at $6 \mathrm{~g} \mathrm{~m}^{-2}$ given as 5-day CIV. The latter ifosfamide schedule was chosen as in multidrug cytotoxic schedules; ifosfamide is frequently administered for 5 days, for example, in combination with cisplatin and etoposide. Additional patients were treated at the MTD of sunitinib with both ifosfamide regimens to get a better insight into the safety profile of the combination and to study PK drug-drug interactions. With regard to the latter, patients enrolled in these dose-expansion cohorts initiated sunitinib at day 8 of the first cycle instead of at day 1, which enables the investigation of the $\mathrm{PK}$ of sunitinib alone. On the basis of the mean half-life of sunitinib $(40-60 \mathrm{~h})$, it was anticipated that steady state of sunitinib levels was reached before initiating the second cycle of ifosfamide. Samples for PK evaluation were collected during the first two treatment cycles. After a protocol amendment because of prolonged neutropenia, granulocyte-colony stimulating factor (pegfilgrastim $6 \mathrm{mg}$ once per cycle) was administered to all patients.

Twelve days before the first administration of study treatment and throughout the whole study, concurrent treatment with known CYP3A4 inhibitors or inducers was not allowed.

Using the Common Terminology Criteria for adverse events (CTCAE), version 3.0, dose-limiting toxicity (DLT) was defined as the following toxicity during the first treatment cycle: grade 4 neutropenia $\geqslant 7$ days, febrile neutropenia, grade 4 thrombocytopenia, serum creatinine $\geqslant 2 \times \mathrm{ULN}$, and any drug-related grade 3 or 4 non-haematological toxicity excluding the following events: nausea and vomiting without optimal supportive care, grade 3 fatigue $<7$ days, and hypertension not refractory to anti-hypertensive medication. If patients developed a systolic blood pressure $>160 \mathrm{~mm} \mathrm{Hg}$, a diastolic blood pressure $>100 \mathrm{~mm} \mathrm{Hg}$, or an increase in diastolic blood pressure $>20 \mathrm{~mm} \mathrm{Hg}$, which (despite anti-hypertensive medication with an ACE inhibitor and a calcium-channel blocker) was not adequately controlled within 2 weeks, treatment with sunitinib was stopped. In case of grade 4 hypertension, sunitinib was also discontinued. A dose delay or interruption for longer than 2 weeks was also classified as DLT.

A classic $3+3$ design was applied, implying that if a DLT was observed in one patient, three additional patients were recruited at that dose level, with the dose level escalating if no further DLT occurred at that level. If a DLT was observed in $\geqslant 2$ patients in a cohort, it could be concluded that the MTD had been exceeded. Maximally tolerated dose was defined as the highest dose level with a DLT incidence of $<33 \%$.

Before commencing each ifosfamide cycle, patients had to have neutrophils $\geqslant 1.5 \times 10^{9}$ cells per 1 and platelets $\geqslant 100 \times 10^{9}$ cells per l. If a patient experienced an ifosfamide-related DLT, the dose of ifosfamide was reduced by $25 \%$. A dose reduction of more than $50 \%$ of the initial ifosfamide dose was not allowed. In those patients experiencing a DLT related to sunitinib, sunitinib was withheld for a maximum of 2 weeks. If toxicity resolved to grade 0 or 1 , continuation at the next lower dose cohort level was allowed for the subsequent courses. Patients were treated for a maximum of six ifosfamide cycles. Those patients who experienced a benefit from the combination of sunitinib and ifosfamide were allowed to continue treatment with sunitinib monotherapy. Treatment was continued until disease progression or unacceptable toxicity.

\section{PK sampling and analysis}

In patients enrolled in the expansion cohorts, blood samples for PK analysis were collected.

For ifosfamide and its most important metabolites, 2-dechloroethyl-ifosfamide, 3-dechloroethyl-ifosfamide, and 4-hydroxy-ifosfamide blood samples were collected in the presence of lithium heparin as anti-coagulant before infusion and 3,6,10, and $24 \mathrm{~h}$ after the start of ifosfamide infusion, and thereafter every $12 \mathrm{~h}$ until the end of infusion, before the end of infusion and $1,3,6,12$, and $24 \mathrm{~h}$ after the end of infusion during the first two treatment cycles. Blood samples were centrifuged within $15 \mathrm{~min}$ after collection for $10 \mathrm{~min}$ at $3000 \mathrm{~g}$ at $4{ }^{\circ} \mathrm{C}$. Subsequently, an aliquot of exactly $1 \mathrm{ml}$ of the plasma supernatant was transferred into a vial containing $100 \mu \mathrm{l}$ of a $2 \mathrm{M}$ semicarbazide solution and was stored at $<-70^{\circ} \mathrm{C}$ until analysis of 4-hydroxy-ifosfamide. The remaining plasma was stored at $<-70^{\circ} \mathrm{C}$, without any additive, until the simultaneous analysis of ifosfamide and its 2-dechloroethyl and 3-dechloroethyl metabolites. Ifosfamide and the 2-dechloroethyl and 3-dechloroethyl metabolites were simultaneously quantitated by a validated liquid chromatography tandem triple quadrupole mass spectrometry (LC-MS/MS) assay. Analytes were extracted by liquid-liquid extraction from $10 \mu \mathrm{l}$ aliquots of plasma with cyclofosfamide as internal standard. For 4-hydroxy-ifosfamide, a separate LC-MS/MS method was developed and validated. Aliquots of $50 \mu \mathrm{l}$ of semicarbazide-stabilised plasma were extracted by liquid-liquid extraction with the same internal standard. Peak area ratios were a function of the concentration from 50.0 to $5000 \mathrm{ng} \mathrm{ml}^{-1}$ for all analytes, with the within and between-run precisions $\leqslant 4.9$ and $\leqslant 5.2 \%$, respectively, and the average accuracy ranging from 90.0 to $105.4 \%$. Individual $\mathrm{PK}$ parameters for ifosfamide, 2-dechloroethyl-ifosfamide and 3-dechloroethylifosfamide, and 4-hydroxy-ifosfamide were estimated using noncompartmental analysis (1/y weighting factor) using the software programme WinNonLin 5.0 (Pharsight, Mountain View, CA, USA).

For the analysis of sunitinib and its active metabolite SU12662, blood samples were taken before dosing every 3-4 days and every day during the second ifosfamide cycle. Blood samples were centrifuged within $15 \mathrm{~min}$ after collection for $10 \mathrm{~min}$ at $3000 \mathrm{~g}$ 
at $4{ }^{\circ} \mathrm{C}$. The plasma was stored at $<-70^{\circ} \mathrm{C}$, in tubes wrapped with aluminium foil, until the simultaneous analysis of sunitinib and SU12662, as recently published (De Bruijn et al, 2010).

\section{Statistical data analysis}

Statistical analysis, using software package SPSS (version 15 (Softonic International, San Francisco, CA, USA)), of the changes in sunitinib concentration was carried out by Wilcoxon signed ranks test using the pre-ifosfamide sunitinib concentration as comparator. Changes in ifosfamide concentration have been evaluated using the same test. Correlation of the auto-induction rate of ifosfamide and changes in sunitinib concentrations was carried out by the Pearson's correlation test.

\section{Biomarker analysis}

Circulating endothelial cell (CEC) enumeration, considered to reflect vascular damage, was determined using the CellSearch system (Veridex, LCC, Raritan, NJ, USA) (Rowand et al, 2007). Plasma concentrations of VEGF and soluble VEGFR2 (sVEGFR2) were determined using ELISA (R\&D Systems, Minneapolis, MN, USA) according to the manufacturer's instructions.

\section{RESULTS}

\section{Dose escalation, MTD, and dose intensity}

In total, 32 patients were enrolled (Table 1). At the first dose level (sunitinib $12.5 \mathrm{mg}$ and ifosfamide $9 \mathrm{~g} \mathrm{~m}^{-2}$ for 3 days), a DLT occurred in two out of six patients; both experienced a prolonged, uncomplicated neutropenia ( $>7$ days). This was pre-specified as exceeding the MTD. The protocol was amended and subsequent

Table I Demographics and baseline characteristics (in numbers (\%) if not otherwise specified)

\begin{tabular}{lc}
\hline Age (median, years) & 53 (range 29-74) \\
Gender & \\
Male & $16(50)$ \\
Female & $16(50)$ \\
WHO performance status & \\
0 & \\
I & $11(34)$ \\
Tumour type & $21(66)$ \\
Sarcoma & \\
Chondrosarcoma & \\
Leiomyosarcoma & $15(47)$ \\
Liposarcoma & $2(6)$ \\
Ewing sarcoma & $2(6)$ \\
Pleomorphic sarcoma & $2(6)$ \\
Other sarcoma types & $2(6)$ \\
Carcinoma of unknown primary tumour & $2(6)$ \\
Neuroendocrine carcinoma & $5(16)$ \\
Non-small-cell lung cancer & $3(9)$ \\
Melanoma (uveal and mucosal) & $2(6)$ \\
Miscellaneous & $2(6)$ \\
Previous non-hormonal systemic anticancer treatment & $2(6)$ \\
0 & $8(26)$ \\
I & \\
2 & \\
3 & $5(16)$ \\
4 & $18(56)$ \\
\hline & $5(16)$ \\
Abbration WW & $2(6)$ \\
& $2(6)$ \\
\hline
\end{tabular}

Abbreviation: $\mathrm{WHO}=$ World Health Organisation. ${ }^{a}$ Bile duct carcinoma, breast cancer, adenoid cystic carcinoma, endometrial cancer, urothelial cancer, pancreatic cancer, chordoma and cervical carcinoma; for all the cases $n=1$. patients were treated with pegfilgrastim. The first dose level was repeated and no DLTs were observed. However, in the subsequent $25 \mathrm{mg}$ sunitinib cohort, three DLTs occurred in five patients (two grade 4 febrile neutropenia; in one patient, hypertension accompanied by chest pain), indicating that the MTD was exceeded, rendering $12.5 \mathrm{mg}$ sunitinib plus ifosfamide $9 \mathrm{~g} \mathrm{~m}^{-2}$ for 3 days combined with pegfilgrastim to be the MTD. After confirming the tolerability at this dose level (in total, one DLT in nine patients: grade 3 febrile neutropenia), the safety of this sunitinib dose was tested with the second ifosfamide regimen $\left(6 \mathrm{~g} \mathrm{~m}^{-2}\right.$ for 5 days), also supported with pegfilgrastim. In nine patients, one DLT was observed (grade 3 ifosfamide-induced encephalopathy).

The dose intensities of sunitinib at the MTD were 93 and $98 \%$ during combination treatment with the 3-day and 5-day schedule, respectively. A median of 4 and 3.5 cycles of ifosfamide was administered, resulting in an ifosfamide dose intensity of 93 and $96 \%$ for the 3-day and 5-day schedule, respectively.

\section{Toxicity}

The non-haematological toxicity of sunitinib and ifosfamide was mainly grade 1-2 toxicity. Haematological toxicity was more pronounced, as grade 3-4 neutropenia was observed in 19 patients (59\%), resulting in one or more episodes of febrile neutropenia in 7 patients $(22 \%)$ (Table 2). At the MTD, in almost every patient in the 3-day schedule (eight out of nine; $89 \%$ ), grade 3-4 neutropenia, and in four patients febrile neutropenia, occurred during combination therapy, whereas only three out nine $(33 \%)$ patients treated with the 5-day schedule had grade 3-4 neutropenia, and no episodes of febrile neutropenia were observed.

\section{Pharmacokinetics}

Pharmacokinetic data of sunitinib and ifosfamide were obtained in six patients per ifosfamide schedule at the MTD. Plasma concentration time curves of ifosfamide, 2-dechloroethyl-ifosfamide,

Table 2 Adverse events during combination therapy of sunitinib and ifosfamide (number of patients (\%))

\begin{tabular}{|c|c|c|c|c|}
\hline & \multicolumn{2}{|c|}{$\begin{array}{c}\text { First cycle adverse } \\
\text { events }\end{array}$} & \multicolumn{2}{|c|}{$\begin{array}{l}\text { Adverse events at all } \\
\text { combination cycles }\end{array}$} \\
\hline & $\begin{array}{c}\text { All } \\
\text { grades }\end{array}$ & Grade $3 / 4$ & All grades & Grade $3 / 4$ \\
\hline \multicolumn{5}{|l|}{ Haematological toxicity } \\
\hline Anaemia & 28 & 0 & 31 & 2 \\
\hline Thrombocytopenia & 19 & 4 & 22 & 9 \\
\hline Neutropenia & 14 & 10 & 21 & 19 \\
\hline Febrile neutropenia & 3 & 3 & 7 & 7 \\
\hline \multicolumn{5}{|l|}{ Non-haematological toxicity } \\
\hline Fatigue & 24 & 2 & 29 & 4 \\
\hline Nausea & 20 & 0 & 24 & 0 \\
\hline Constipation & 20 & 0 & 24 & 0 \\
\hline ALAT and/or ASAT & 20 & 0 & 21 & 0 \\
\hline Alopecia & 19 & 0 & 26 & 0 \\
\hline Vomiting & 14 & 0 & 19 & 0 \\
\hline Creatinine/renal & 9 & 0 & 12 & 1 \\
\hline Hypo-/hyperkalaemia & $5 / 1$ & $1 / 0$ & $5 / 1$ & $2 / 0$ \\
\hline Bilirubin & 5 & 0 & 6 & 0 \\
\hline Diarrhoea & 4 & 0 & 5 & 0 \\
\hline Pyrosis & 3 & 0 & 5 & 0 \\
\hline TSH increase/decrease & $2 / 2$ & 0 & $2 / 2$ & 0 \\
\hline Neurotoxicity & 2 & 1 & 8 & 1 \\
\hline Anorexia & 2 & 0 & 11 & 1 \\
\hline Haemorrhage & 1 & 1 & I & 1 \\
\hline
\end{tabular}

Abbreviations: $\mathrm{ALAT}=$ alanine transaminase; $\mathrm{ASAT}=$ aspartate aminotransferase; $\mathrm{TSH}=$ thyroid-stimulating hormone. 

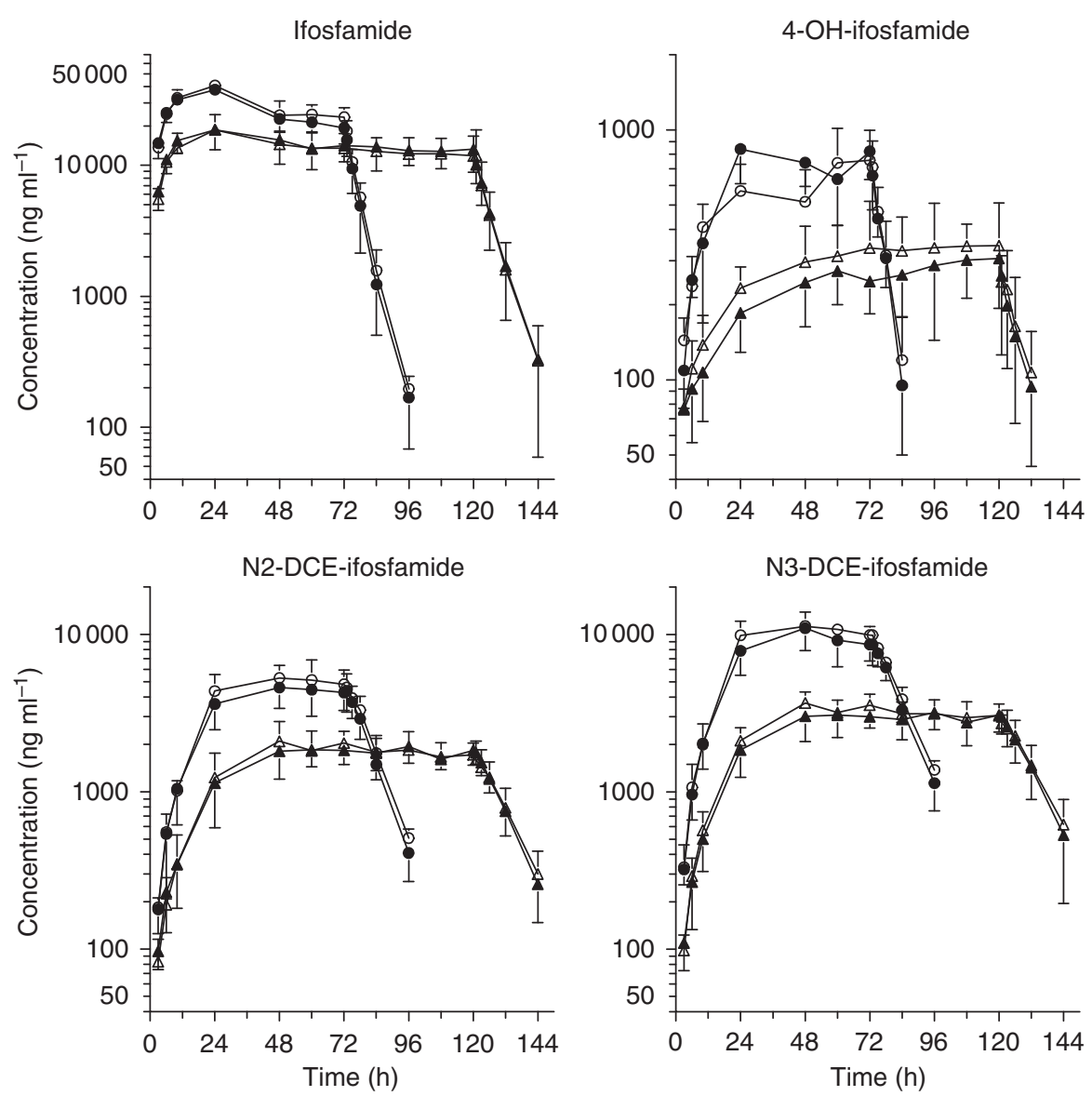

Figure I Mean concentrations (plus s.d.) of ifosfamide, 4-hydroxy-ifosfamide (4-OH-ifosfamide), 2-dechloroethyl-ifosfamide (N2-DCE-ifosfamide), and 3-dechloroethyl-ifosfamide (N3-DCE-ifosfamide) administered alone (open red symbols: bars up) or in combination with sunitinib (closed blue symbols, bars down) during the 3-day (open and closed circles) and 5-day (open and closed triangles) continuous infusions (The colour reproduction of this figure is available on the html full text version of the manuscript).

3-dechloroethyl-ifosfamide, and 4-hydroy-ifosfamide were not affected by sunitinib (Figure 1). Ifosfamide concentrations were significantly lower at $48 \mathrm{~h}\left(\mathrm{C}_{48 \mathrm{~h}}\right)$ compared with $24 \mathrm{~h}\left(\mathrm{C}_{24 \mathrm{~h}}\right)$ in the 3 -day and 5 -day schedule $(40 \%$ and $17 \%$, respectively; both $P=0.028)$, in line with the known ifosfamide capacity to induce its own CYP3A-mediated metabolism (Boddy et al, 1995).

Sunitinib trough concentrations decreased during ifosfamide infusions during the 3-day and 5-day schedule (Figure 2 upper graphs). As presented in Figure 3 (upper panel), trough concentrations were significantly lower at 1,2 , and 3 days $(P<0.05)$ after the start of ifosfamide infusion in the 3-day schedule, whereas during the 5 -day schedule, the decrease was less pronounced, not reaching statistical significance. The decrease in sunitinib was paralleled by an increase in the trough concentrations of its pharmacologically active metabolite SU12662 (Figure 2, lower graphs), which reached statistical significance in both the 3-day and 5-day ifosfamide schedules (Figure 3, middle panel). However, the sum of sunitinib and its pharmacologically active metabolite, SU12662, slightly decreased during the ifosfamide infusions, reaching significance only 1 day after the start of ifosfamide infusion in the 3-day schedule $(P<0.05)$ (Figure 3, lower panel).

The auto-induction rate of ifosfamide, expressed as $\mathrm{C}_{48 \mathrm{~h}} / \mathrm{C}_{24 \mathrm{~h}}$, was correlated to the decrease in sunitinib trough concentrations, expressed as $\mathrm{C}_{\text {day } 2} / \mathrm{C}_{\text {day } 0}\left(R^{2}=0.47 ; P=0.019\right)$ (Figure 4).

\section{Biomarker analysis}

Circulating endothelial cell enumeration and determination of plasma concentrations of VEGF and sVEGFR2 with serial sampling were successful in 22 patients. A wide range in relative changes in the number of CECs from baseline to post-cycle $2(0.05-35.2)$ and post-cycle $5(0.09-5.2)$ was observed. Relative changes from baseline to post-cycle 2 and post-cycle 5 were widespread for VEGF (0.04-14.1 and 0.25-5.6, respectively) and less outspoken for sVEGFR2 (0.63-1.4 and 0.46-1.1, respectively). No consistent change in time, correlation with dose levels, with the occurrence of febrile neutropenia, or tumour response was observed for VEGF or sVEGFR2 plasma concentrations or number of CECs.

\section{Anti-tumour activity}

All 32 patients were assessable for efficacy. Partial responses were seen during the 3-day schedule in three patients (one patient with adenocarcinoma of unknown primary, one patient with a sarcoma NOS, and one patient with a liposarcoma). Disease stabilisation was observed in 17 patients in whom a prolonged ( $>3$ months) disease stabilisation was observed in 9 patients ( 7 out of 23 patients during the 3 -day schedule and 2 out of 9 patients during the 5-day schedule). The latter group consisted of patients with uveal melanoma, chordoma, chondrosarcoma, Ewing sarcoma, uterus sarcoma, endometrial carcinoma, small-cell neuroendocrine carcinoma, non-small-cell lung cancer, and carcinoma of unknown primary (all entities $n=1$ ).

\section{DISCUSSION}

This is one of the first full reports on a combination of sunitinib with a conventional cytotoxic agent and the first on the 


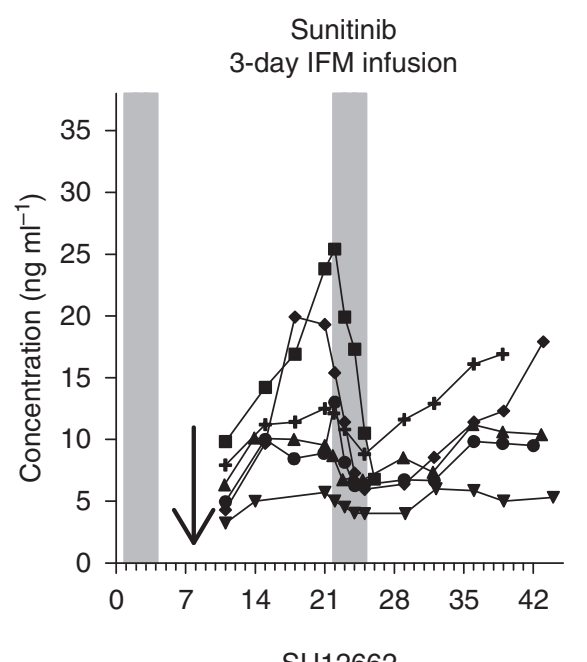

SU12662

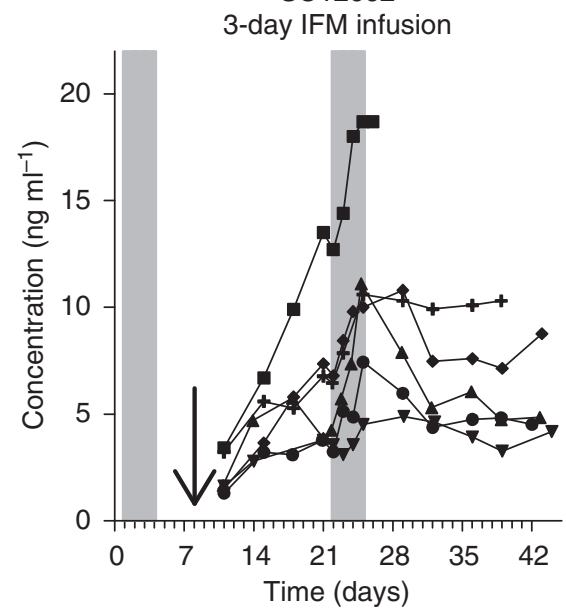

Sunitinib

5-day IFM infusion

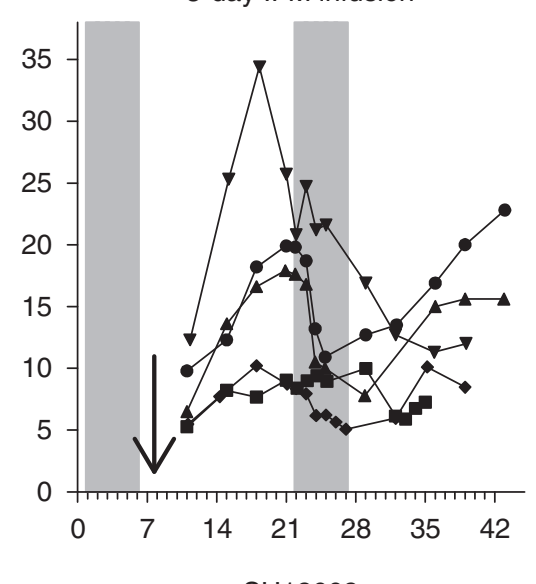

SU12662

5-day IFM infusion

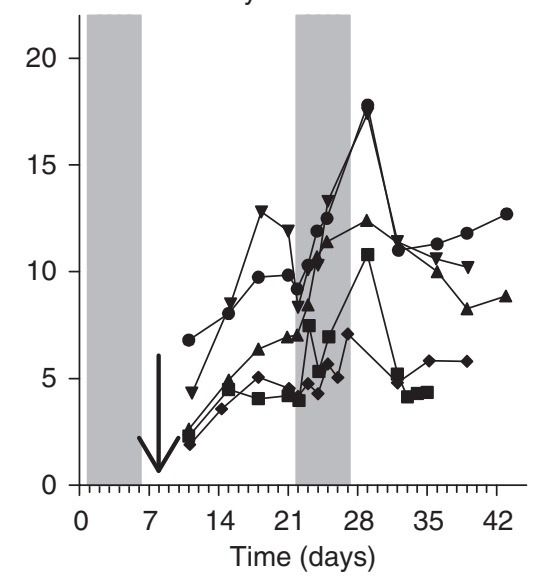

Figure 2 Individual plasma concentration time curves of sunitinib in combination with 3-day $(n=6)$ and 5-day ( $n=5)$ ifosfamide schedule (upper graphs) and of SUI 2662 in combination with 3-day $(n=6)$ and 5-day $(n=5)$ ifosfamide schedule (lower graphs). One patient in the 5-day schedule has not been included in the figure as cycle two was delayed by 2 weeks. Bars represent the ifosfamide infusion schedules and arrows the first sunitinib administrations.

combination of ifosfamide with a VEGFR-inhibiting TKI. Combined with ifosfamide at $9 \mathrm{~g} \mathrm{~m}^{-2}$ given continuously over 3 days, it seemed that the MTD of sunitinib is mainly determined by neutropenia-related events. With growth factor support, sunitinib at the lowest evaluated dose of $12.5 \mathrm{mg}$ daily was feasible in combination with ifosfamide at $9 \mathrm{~g} \mathrm{~m}^{-2}$ for 3 days and at $6 \mathrm{~g} \mathrm{~m}^{-2}$ for 5 days. Importantly, our data on the occurrence of neutropenia-related adverse events should be appreciated in the context of toxicity from ifosfamide monotherapy. Ifosfamide given at $9 \mathrm{~g} \mathrm{~m}^{-2}$ for 3 days, as an established treatment schedule, has an observed incidence in a phase III trial of $62.7 \%$ grade 3-4 neutropenia when given as first-line therapy. In addition, during a mean number 3.7 cycles of therapy, $19.6 \%$ of the patients encountered febrile neutropenia (Lorigan et al, 2007). Furthermore, sunitinib administered as a single agent produces grade 3-4 neutropenia in $12 \%$ of patients (Motzer et al, 2007). Given these figures, it comes as no surprise that neutropeniarelated adverse events were the determining toxicity for the MTD of the combination. On the basis of the findings in this study, it is clear that the occurrence of neutropenia-related events is not attributable to changes in drug exposure.

Recently, several trials on sunitinib combined with conventional cytotoxic agents have been reported, although only in abstract form. The majority of the tested combinations also yielded a high incidence of neutropenia-related events, which were frequently dose-limiting toxicities. This held true for the combinations of sunitinib with docetaxel, irinotecan, FOLFIRI, carboplatin/ paclitaxel, or gemcitabine/cisplatin, preventing sunitinib from being administered at full single-agent doses (Reck et al, 2007; Robert et al, 2007; Boven et al, 2008; Mariani et al, 2008; Starling et al, 2008; Cardoso et al, 2009; Friedman et al, 2009; Heath et al, 2009; Zurita et al, 2009). The only two combinations not hampered by neutropenia-related adverse events were sunitinib with capecitabine or with gemcitabine (Michaelson et al, 2008; Royce et al, 2008).

What becomes apparent from these studies is the fact that the schedule in which sunitinib is administered is likely to largely impact the tolerability of combinations in terms of neutropeniarelated events. The recommended dose of sunitinib as a single agent was initially reported as $50 \mathrm{mg}$ administered daily for 28 days every 6 weeks. However, we choose to administer sunitinib continuously. Evidence accumulates that persistent inhibition of the VEGF pathway might be advantageous over intermittent dosing, although both dosing schedules have not been directly compared yet. For combinations, however, toxicity might be more pronounced using sunitinib continuously rather than intermittently, as recovery from toxicity induced by the cytotoxic agent might be hampered in the presence of sunitinib. Accordingly, preliminary data on the combination of sunitinib with FOLFIRI suggest that continuous dosing of sunitinib was not feasible 

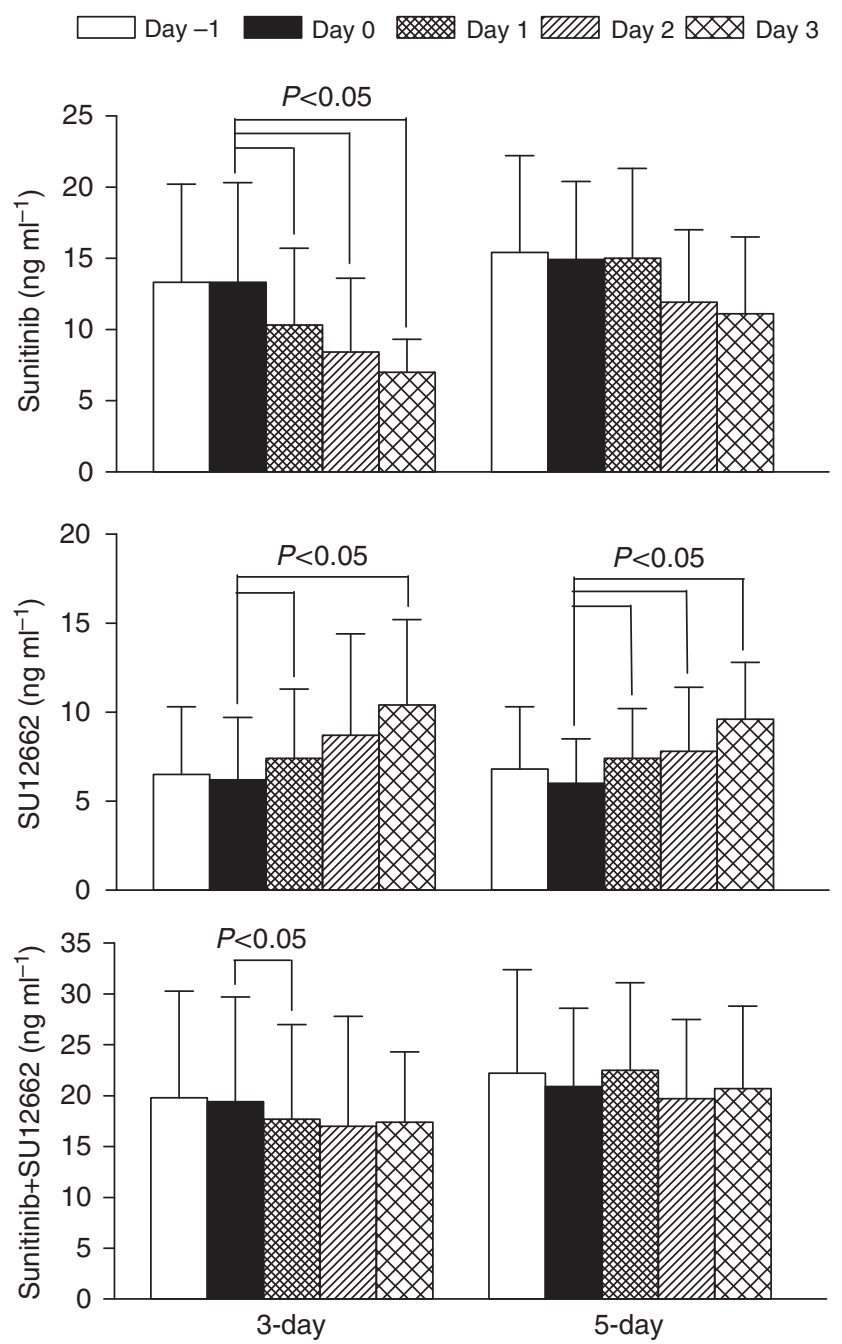

Figure 3 Absolute concentration of sunitinib (upper panel), SUI 2262 (middle panel), and the sum of sunitinib plus SUI2662 (lower panel) in patients after the 3-day and 5-day continuous infusions of ifosfamide. $P$-values (Wilcoxon signed rank test) of significant lower or higher relative trough concentrations compared with the trough concentrations observed before the start of ifosfamide infusions (that is, day 0 , lime bars) are presented. Day - I (brown bars) is the sample taken the day before the start of ifosfamide infusion and days I (red bars), 2 (blue bars), and 3 (rose bars) are the samples taken 24,48 , and $72 \mathrm{~h}$ after the start of ifosfamide infusions, respectively. Data are presented as the mean \pm s.d. of six observations; $n=5$ for day 2 in the 3 -day schedule (The colour reproduction of this figure is available on the $\mathrm{html}$ full text version of the manuscript).

because of neutropenia, whereas in contrast, sunitinib administered in a 4-week on, 2-week off schedule could be applied at a dose of $37.5 \mathrm{mg}$ in combination with FOLFIRI (Starling et al, 2008). From a mechanism of action point of view, the leastdesirable administration schedule of sunitinib might be best combinable with chemotherapy. In contrast, sunitinib continuously administered, as well as administered in the 4-week on 2-week off schedule in combination with capecitabine, was tolerated at the same sunitinib dose ( $37.5 \mathrm{mg}$ per day); however, as mentioned before, neutropenia was not a major issue in that specific combination (Royce et al, 2008).

Besides the neutropenia-related adverse events, the combination of (a relatively low dose of) sunitinib, using continuous administration, and ifosfamide was well tolerated (Table 2).

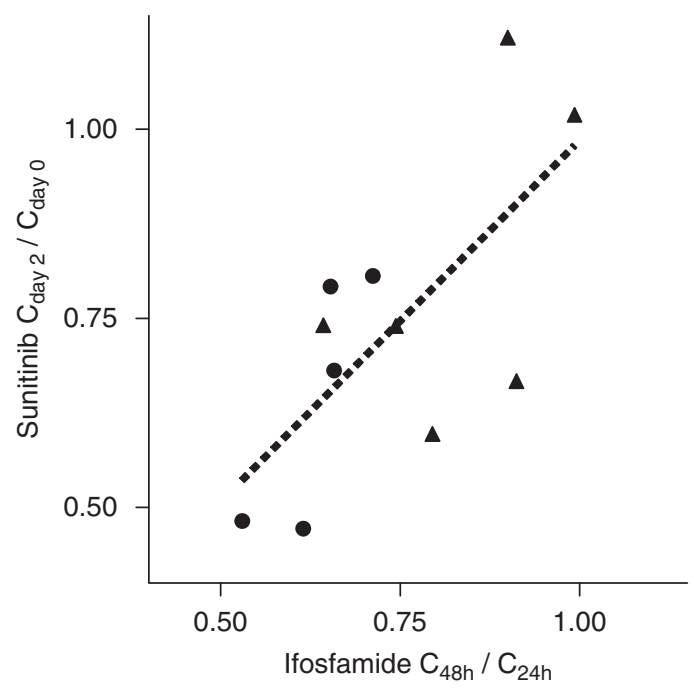

Figure 4 Linear relationship $\left(R^{2}=0.47 ; P=0.019\right)$ between autoinduction of ifosfamide and decrease in sunitinib trough concentrations during the 3-day (closed red circles) and 5-day (closed blue triangles) ifosfamide infusions (The colour reproduction of this figure is available on the html full text version of the manuscript).

Assessing the PK of both drugs, a clear influence of ifosfamide on the PK of sunitinib was observed. This interaction resulted in a decreased systemic exposure to sunitinib and an increased exposure to its active metabolite, SU12662. Although the exact contribution of the active metabolite SU12662 to the toxicity and efficacy pattern of sunitinib in humans is unknown, preclinical data point towards equipotent inhibitory capacities (Baratte et al, 2004). Ifosfamide is a potent inhibitor of CYP3A, the enzyme mainly responsible for conversion of sunitinib into SU12662 (Faivre et al, 2006). This observed drug-drug interaction is in line with findings in healthy subjects showing a decreased systemic exposure to sunitinib when concomitantly treated with the potent CYP3A inducer rifampicin (Bello et al, 2005) and an increased exposure in the presence of ketoconazole, a potent CYP3A inhibitor (Washington et al, 2003). Pharmacokinetic drug-drug interactions have been studied combining sunitinib with gemcitabine, capecitabine, paclitaxel, and docetaxel with or without trastuzumab, but no relevant $\mathrm{PK}$ interactions were observed in these studies (Mariani et al, 2008; Michaelson et al, 2008; Royce et al, 2008; Cardoso et al, 2009).

As a relatively low dose of sunitinib in combination with a standard dose of ifosfamide already results in DLT, which cannot be explained by PK interactions, synergy in toxicity seems most likely. An explanatory hypothesis for the occurrence of the described neutropenia-related events while combining ifosfamide with sunitinib might consist of a dual hit. Initially, neutrophils decrease because of the administration of ifosfamide. The physiological response of mobilisation and proliferation of haematopoietic progenitor cells to restore the neutrophil count is hampered because of inhibition by sunitinib of tyrosine kinases involved in haematopoietic progenitor cell survival and proliferation, such as Flt3 and colony-stimulating factor receptor (CSF-1R).

Importantly, a multiple drug combination should not be discarded as a result of its low combinability because of synergy in toxicity alone, as this synergistic interaction may also occur at tumour cell level.

As PD parameters, CEC numbers and plasma concentrations of VEGF and sVEGFR2 were assessed. Alterations in CEC numbers is likely to reflect vascular damage (Strijbos et al, 2008), but no consistent changes in CEC numbers could be seen, either during therapy or between the two sunitinib doses explored. In contrast to 
monotherapy with sunitinib (Deprimo et al, 2007), no consistent pattern in the changes in plasma concentrations of VEGF and sVEGFR2 was observed.

In conclusion, the MTD of daily sunitinib combined with continuously infused ifosfamide $\left(9 \mathrm{~g} \mathrm{~m}^{-2}\right.$ for 3 days) supported by pegfilgrastim is $12.5 \mathrm{mg}$. The same dose of sunitinib is feasible using an ifosfamide schedule of 5 days (total dose per cycle $6 \mathrm{~g} \mathrm{~m}^{-2}$ ). Concomitant treatment with ifosfamide significantly decreased the systemic exposure to sunitinib, whereas the exposure to its active metabolite, SU12662, increased because of CYP3A induction. As PK interactions cannot explain the fact that ifosfamide can be combined safely only with relatively low sunitinib doses, synergy in toxicity is likely. Whether this holds true for anti-tumour activity needs to be determined, and it is

\section{REFERENCES}

Baratte S, Sarati S, Frigerio E, James CA, Ye C, Zhang Q (2004) Quantitation of SU11248, an oral multi-target tyrosine kinase inhibitor, and its metabolite in monkey tissues by liquid chromatography with tandem mass spectrometry following semi-automated liquid-liquid extraction. J Chromatogr A 1024: 87-94

Bello C, Houk B, Sherman L, Misbah S, Sarapa N, Smeraglia J, Haung X (2005) Effect of rifampin on the pharmacokinetics of SU11248 in healthy volunteers. J Clin Oncol 23: 3078

Boddy AV, Cole M, Pearson AD, Idle JR (1995) The kinetics of the auto-induction of ifosfamide metabolism during continuous infusion. Cancer Chemother Pharmacol 36: 53-60

Boucher Y, Jain RK (1992) Microvascular pressure is the principal driving force for interstitial hypertension in solid tumors: implications for vascular collapse. Cancer Res 52: 5110-5114

Boven E, Massard C, Armand JP, Tiller C, Hartog V, Brega NM, Contouriotis AM, Law CNJ, Soria J (2008) Phase I, dose-finding study of sunitinib in combination with irinotecan in patients with advanced solid tumors. Ann Oncol 19: viii156

Cardoso F, Canon JL, Amadori D, Dirix L, Villa E, Aldrighetti D, Machiels JP, Verkh L, Kern K, Giorgetti C (2009) Sunitinib plus docetaxel and trastuzumab as first-line therapy for HER2+ advanced breast cancer. Eur J Cancer Suppl 7: 2835075

De Bruijn P, Sleijfer S, Lam MH, Mathijssen RH, Wiemer EA, Loos W] (2010) Bioanalytical method for the quantification of sunitinib and its n-desethyl metabolite SU12662 in human plasma by ultra performance liquid chromatography/tandem triple-quadrupole mass spectrometry. J Pharm Biomed Anal 51: 934-941

Demetri GD, van Oosterom AT, Garrett CR, Blackstein ME, Shah $\mathrm{MH}$, Verweij J, McArthur G, Judson IR, Heinrich MC, Morgan JA, Desai J, Fletcher CD, George S, Bello CL, Huang X, Baum CM, Casali PG (2006) Efficacy and safety of sunitinib in patients with advanced gastrointestinal stromal tumour after failure of imatinib: a randomised controlled trial. Lancet 368: $1329-1338$

Deprimo SE, Bello CL, Smeraglia J, Baum CM, Spinella D, Rini BI, Michaelson MD, Motzer RJ (2007) Circulating protein biomarkers of pharmacodynamic activity of sunitinib in patients with metastatic renal cell carcinoma: modulation of VEGF and VEGF-related proteins. J Transl Med 5: 32

Dias S, Shmelkov SV, Lam G, Rafii S (2002) VEGF(165) promotes survival of leukemic cells by Hsp90-mediated induction of Bcl-2 expression and apoptosis inhibition. Blood 99: 2532-2540

Faivre S, Delbaldo C, Vera K, Robert C, Lozahic S, Lassau N, Bello C, Deprimo S, Brega N, Massimini G, Armand JP, Scigalla P, Raymond E (2006) Safety, pharmacokinetic, and antitumor activity of SU11248, a novel oral multitarget tyrosine kinase inhibitor, in patients with cancer. J Clin Oncol 24: 25 - 35

Friedman HS, Vredenburgh JJ, Desjarddins A, Janney DE, Peters KB, Friedman AH, Gururangan S, Reardon A (2009) A phase I study of sunitinib plus irinotecan in the treatment of patients with recurrent glioma. J Clin Oncol 27: e13024

Heath EI, Blumenschein GR, Cohen RB, LoRusso PM, LoConte NK, Kim S, Chao R, Wilding G (2009) Sunitinib in combination with paclitaxel and carboplatin in patients with advanced solid tumors; updated phase I study results. J Clin Oncol 27: e14509 particularly attractive to explore this further in tumour types against which both sunitinib and ifosfamide as monotherapy exhibit anti-tumour activity, such as soft tissue sarcomas, as well as lung and breast cancer.

\section{ACKNOWLEDGEMENTS}

The assistance of Peter de Bruijn, Cor Lamers, Jaco Kraan, Patricia de Vos, Merlijn Cranendonk, Inge Ghobadi MoghaddamHelmantel, Mei-Ho Lam, and Jan Ouwerkerk is kindly acknowledged. This is an investigator-initiated trial financially supported by Pfizer Netherlands BV.
Heldin $\mathrm{CH}$, Rubin K, Pietras K, Ostman A (2004) High interstitial fluid pressure - an obstacle in cancer therapy. Nat Rev Cancer 4: 806-813

Lorigan P, Verweij J, Papai Z, Rodenhuis S, Le Cesne A, Leahy MG, Radford JA, Van Glabbeke MM, Kirkpatrick A, Hogendoorn PC, Blay JY, European Organisation for Research and Treatment of Cancer Soft Tissue and Bone Sarcoma Group Study (2007) Phase III trial of two investigational schedules of ifosfamide compared with standard-dose doxorubicin in advanced or metastatic soft tissue sarcoma: a European Organisation for Research and Treatment of Cancer Soft Tissue and Bone Sarcoma Group Study. J Clin Oncol 25: $3144-3150$

Mariani G, Cardoso F, Besse-Hammer T, Vigano L, Liljegren A, Verkh L, Huang X, Giorgetii C, Bergh J, Gianni L (2008) Sequential administration of sunitinib and docetaxel in women with advanced breast cancer: an exploratory evaluation. J Clin Oncol 26: 18s

Michaelson MD, Schwarzberg A, Ryan DP, McDermott D, Shapiro GI, Tye L, Chen I, Selaru P, Wang E, Zhu A (2008) A phase I dose-finding study of sunitinib (SU) in combination with gemcitabine $(\mathrm{G})$ in patients (pts) with advanced solid tumors. Ann Oncol 19: viii156

Motzer RJ, Hutson TE, Tomczak P, Michaelson MD, Bukowski RM, Rixe O, Oudard S, Negrier S, Szczylik C, Kim ST, Chen I, Bycott PW, Baum CM, Figlin RA (2007) Sunitinib versus interferon alfa in metastatic renal-cell carcinoma. N Engl J Med 356: 115 - 124

Reck M, Frickhofen N, Gatzemeier U, Fuhr H, Lanzalon S, Lechuga J, Wang E, Chao R, Felip E (2007) A phase I dose escalation study of sunitinib in combination with gemcitabine + cisplatin for advanced non-small cell lung cancer (NSCLC). $J$ Clin Oncol 25: 18s

Robert F, Sandler A, Schiller JH, Ilagan J, Vermeulen W, Harper K, Liu G, Tye L, Chao R, Traynor A (2007) A phase I dose-escalation and pharmacokinetic study of sunitinib plus docetaxel in patients with advanced solid tumors. I Clin Oncol 25: 18s

Riedel F, Götte K, Goessler U, Sadick H, Hörmann K (2004) Targeting chemotherapy-induced VEGF up-regulation by VEGF antisense oligonucleotides in HNSCC cell lines. Anticancer Res 24: 2179-2183

Rowand JL, Martin G, Doyle GV, Miller MC, Pierce MS, Connelly MC, Rao C, Terstappen LW (2007) Endothelial cells in peripheral blood of healthy subjects and patients with metastatic carcinomas. Cytometry $A$ 71: $105-113$

Royce M, Chiorean EG, Sweeney C, Verschraegen C, Lee FC, Jones S, Rabinowitz I, Bello A, Chao R, Burris H (2008) A phase I dose-finding study of sunitinib in combination with capecitabine in patients with advanced solid tumors. Breast Cancer Symposium Proceedings. 169

Shaked Y, Henke E, Roodhart JM, Mancuso P, Langenberg MH, Colleoni M, Daenen LG, Man S, Xu P, Emmenegger U, Tang T, Zhu Z, Witte L, Strieter RM, Bertolini F, Voest EE, Benezra R, Kerbel RS (2008) Rapid chemotherapy-induced acute endothelial progenitor cell mobilization: implications for antiangiogenic drugs as chemosensitizing agents. Cancer Cell 14: 263-273

Simakajornboon N, Szerlip NJ, Gozal E, Anonetapipat JW, Gozal D (2001) In vivo PDGF beta receptor activation in the dorsocaudal brainstem of the rat prevents hypoxia-induced apoptosis via activation of Akt and BAD. Brain Res 895: $111-118$

Starling N, Vázquez F, Cunningham D, Chau I, Ramos FJ, Saunders MP, Iveson TJ, Verkh L, Tursi J, Carrato A (2008) Phase I study to evaluate the safety and efficacy of sunitinib in combination with FOLFIRI 
in treatment-naïve metastatic colorectal cancer (mCRC). J Clin Oncol 26: $18 \mathrm{~s}$

Strijbos MH, Gratama JW, Kraan J, Lamers CH, den Bakker MA, Sleijfer S (2008) Circulating endothelial cells in oncology: pitfalls and promises. Br J Cancer 98: 1731-1735

Therasse P, Arbuck SG, Eisenhauer EA, Wanders J, Kaplan RS, Rubinstein L, Verweij J, Van Glabbeke M, van Oosterom AT, Christian MC, Gwyther SG (2000) New guidelines to evaluate the response to treatment in solid tumors. European Organization for Research and Treatment of Cancer, National Cancer Institute of the United States, National Cancer Institute of Canada. J Natl Cancer Inst 92: 205-216

Tran J, Master Z, Yu JL, Rak JL, Dumont DJ, Kerbel RS (2002) A role for survivin in chemoresistance of endothelial cells mediated by VEGF. Proc Natl Acad Sci USA 99: 4349-4354

Verweij J, Casali PG, Zalcberg J, LeCesne A, Reichardt P, Blay JY, Issels R, van Oosterom A, Hogendoorn PC, van Glabbeke M, Bertulli R, Judson I
(2004) Progression-free survival in gastrointestinal tumours with high-dose imatinib: randomised trial. Lancet 364: 1127-1134

Washington C, Eli M, Bello C, Schaaf L, Polasek E, Tan LH, Scigalla P, Sarapa N (2003) The effect of ketoconazole, a potent CYP3A4 inhibitor, on SU011248 pharmacokinetics in Caucasian and Asian Healthy subjects. J Clin Oncol 22: 553

Willett CG, Boucher Y, di Tomaso E, Duda DG, Munn LL, Tong RT, Chung DC, Sahani DV, Kalva SP, Kozin SV, Mino M, Cohen KS, Scadden DT, Hartford AC, Fischman AJ, Clark JW, Ryan DP, Zhu AX, Blaszkowsky LS, Chen HX, Shellito PC, Lauwers GY, Jain RK (2004) Direct evidence that the VEGF-specific antibody bevacizumab has antivascular effects in human rectal cancer. Nat Med 10: 145-147

Zurita AJ, Liu G, Hutson T, Kozloff M, Shore N, Wilding G, Logothetis CJ, Chen I, Chow Maneval E, George D (2009) Sunitinib in combination with docetaxel and prednisone in patients with metastatic hormone-refractory prostate cancer. J Clin Oncol 27: 15s 